Using the analytic center in the feasibility pump

\author{
Daniel Baena Jordi Castro \\ Dept. of Stat. and Operations Research \\ Universitat Politècnica de Catalunya \\ daniel.baena@upc.edu jordi.castro@upc.edu \\ Research Report UPC-DEIO DR 2010-03 \\ August 2010
}

Report available from http://www-eio.upc.es/ ${ }^{\sim}$ jcastro 



\title{
Using the analytic center in the feasibility pump
}

\author{
Daniel Baena ${ }^{a}$, Jordi Castro*a \\ ${ }^{a}$ Dept. of Statistics and Operations Research, Universitat Politècnica de Catalunya, Barcelona, Catalonia, Spain
}

\begin{abstract}
The feasibility pump (FP) [5, 7] has proved to be a successful heuristic for finding feasible solutions of mixed integer linear problems (MILPs). FP was improved in [1] for finding better quality solutions. Briefly, FP alternates between two sequences of points: one of feasible solutions for the relaxed problem (but not integer), and another of integer points (but not feasible for the relaxed problem). Hopefully, the procedure may eventually converge to a feasible and integer solution. Integer points are obtained from the feasible ones by some rounding procedure. This short paper extends FP, such that the integer point is obtained by rounding a point on the (feasible) segment between the computed feasible point and the analytic center for the relaxed linear problem. Since points in the segment are closer (may be even interior) to the convex hull of integer solutions, it may be expected that the rounded point has more chances to become feasible, thus reducing the number of FP iterations. When the selected point to be rounded is the feasible solution of the relaxation (i.e., one of the two end points of the segment), this analytic center FP variant behaves as the standard FP. Computational results show that this variant may be efficient in some MILP instances.
\end{abstract}

Key words: Analytic Center, Interior-point Methods, Mixed-integer Linear Programming, Feasibility Problem, Primal Heuristics

\section{Introduction}

The problem of finding a feasible solution of a generic mixed integer linear problem (MILP) of the form

$$
\begin{array}{cl}
\min _{x} & c^{T} x \\
\text { s. to } & A x=b \\
& x \geq 0 \\
& x_{j} \text { integer } \quad \forall j \in \mathcal{I},
\end{array}
$$

where $\mathrm{A} \in \mathbb{R}^{m x n}, b \in \mathbb{R}^{m}, c \in \mathbb{R}^{n}$ and $\mathcal{I} \subseteq \mathcal{N}=\{1, \ldots, n\}$, is a NP-hard problem. In [5, 7] the authors proposed a new heuristic approach to compute MILP solutions, named the feasibility pump (FP). This heuristic turned out to be successful in finding feasible solutions even for some hard MILP instances. A slight modification of FP was suggested in [1], named the objective feasibility

${ }^{*}$ Corresponding address: Dept. of Statistics and Operations Research, Universitat Politècnica de Catalunya, Campus Nord, Office C5218, Jordi Girona 1-3, 08034 Barcelona, Catalonia, Spain.

Email addresses: daniel . baena@upc.edu (Daniel Baena), jordi .castro@upc.edu (Jordi Castro) 


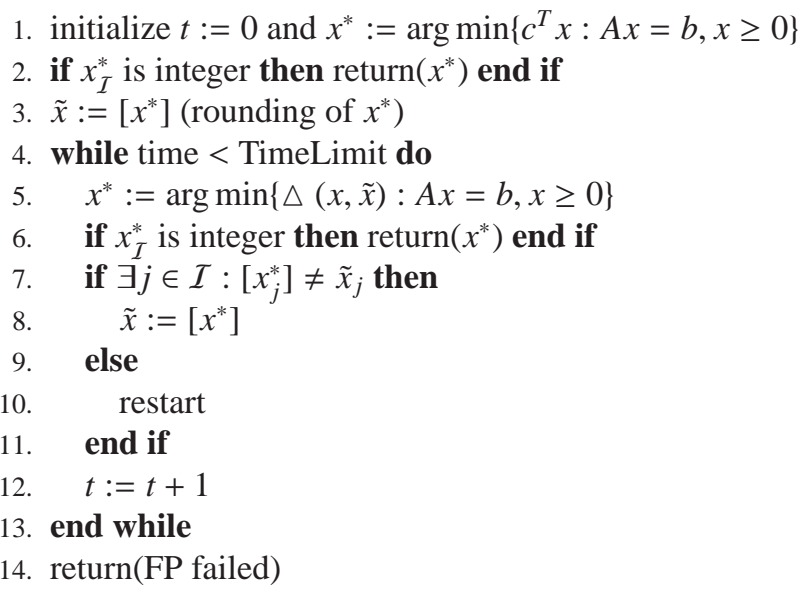

Figure 1: The feasibility pump heuristic (original version)

pump, in order to improve the quality of the solutions in terms of the objective value. The main difference between both versions is that the objective FP, in contrast to the original version, takes the objective function of the MILP into account during the course of the algorithm. FP alternates between feasible (for the linear relaxation of MILP) and integer points, hopefully converging to a feasible integer solution. The integer point is obtained by applying some rounding procedure to the feasible solution. This paper suggests an extension of FP where all the points in a feasible segment are candidates to be rounded. The end points of this segment are the feasible point of the standard FP and some interior point of the polytope of the relaxed problem, the analytic center being the best candidate. When the end point of the segment in the boundary of the polytope is considered for rounding, we obtain the standard FP algorithm. The motivation of this approach is that rounding a point of the segment closer to the analytic center may increase the chances of obtaining an integer point, in some instances, thus reducing the number of FP iterations. Although interior-point methods have been applied in the past in branch-and-bound frameworks for MILP and mixed integer nonlinear problems (MINLP) [3, 4, 11, 12], as far as we know this is the first attempt to apply them to a primal heuristic. The computational results show that, for some instances, taking a point in the interior of the feasible segment may be more effective than the standard end point of the objective FP. A recent version of FP [8] introduced a new improved rounding scheme based on constraint propagation. Although in this work we considered as base code a freely available implementation of the objective FP, the analytic center FP approach could also be used with the above new rounding scheme.

The paper is organized as follows. The remainder of Section 1 reviews the original FP version of $[5,7]$ and the modified objective FP of [1]. Section 2 introduces the analytic center FP variant. Finally, Section 3 reports computational results on a subset of MILP instances from MIPLIB 2003 [2].

\subsection{The original feasibility pump}

The FP heuristic starts by solving the linear programming (LP) relaxation of (1)

$$
\min _{x}\left\{c^{T} x: A x=b, x \geq 0\right\},
$$


and its solution $x^{*}$ is rounded to an integer point $\tilde{x}$, which may be infeasible for (2). The rounding $\tilde{x}$ of a given $x^{*}$, denoted as $\tilde{x}=\left[x^{*}\right]$, is obtained by setting $\tilde{x}_{j}=\left[x_{j}^{*}\right]$ if $j \in \mathcal{I}$ and $\tilde{x}_{j}=x_{j}^{*}$ otherwise, where [.] represents scalar rounding to the nearest integer. If $\tilde{x}$ is infeasible, FP finds the closest $x^{*} \in P$, where

$$
P=\left\{x \in \mathbb{R}^{n}: A x=b, x \geq 0\right\}
$$

by solving the following LP

$$
x^{*}=\arg \min \{\triangle(x, \tilde{x}): A x=b, x \geq 0\},
$$

$\triangle(x, \tilde{x})$ being defined (using the $L_{1}$ norm) as

$$
\triangle(x, \tilde{x})=\sum_{j \in \mathcal{I}}\left|x_{j}-\tilde{x}_{j}\right| .
$$

Notice that continuous variables $\tilde{x}_{j}, j \notin \mathcal{I}$, don't play any role. If $\Delta\left(x^{*}, \tilde{x}\right)=0$ then $x_{j}^{*}\left(=\tilde{x}_{j}\right)$ is integer for all $j \in \mathcal{I}$, so $x^{*}$ is a feasible solution for (1). If not, FP finds a new integer point $\tilde{x}$ from $x^{*}$ by rounding. The pair of points $\left(\tilde{x}, x^{*}\right)$ with $\tilde{x}$ integer and $x^{*} \in P$ are iteratively updated at each FP iteration with the aim of reducing as much as possible the distance $\Delta\left(x^{*}, \tilde{x}\right)$. An outline of the FP algorithm is showed in Figure 1. To avoid that the procedure gets stuck at the same sequence of integer and feasible, there is a restart procedure when the previous integer point $\tilde{x}$ is revisited (lines 7-11 of algorithm of Figure 1). In a restart, a random perturbation step is performed.

The FP implementation has three stages. Stage 1 is performed just on the binary variables by relaxing the integrality conditions on the general integer variables. In stage 2 FP takes all integer variables into account. The FP algorithm exits stage 1 and goes to stage 2 when either (a) a feasible point with respect to only the binary variables has been found; (b) the minimum $\triangle\left(x^{*}, \tilde{x}\right)$ was not updated during a certain number of iterations; or (c) the maximum number of iterations was reached. The point $\tilde{x}$ that produced the smallest $\Delta\left(x^{*}, \tilde{x}\right)$ is stored and passed to stage 2 as the initial $\tilde{x}$ point. When FP turns out to be unable to find a feasible solution within the provided time limit, the default procedure of the underlying MILP solver (CPLEX 12 [10] in this work) is started; this is named stage 3 .

\subsection{The modified objective feasibility pump}

According to [1], although the original FP heuristic of $[5,7]$ has proved to be a very successful heuristic for finding feasible solutions of mixed integer programs, the quality of their solutions in terms of objective value tends to be poor. In the original FP algorithm of [5, 7] the objective function of (1) is only used at the beginning of the procedure. The purpose of the objective FP [1] is, instead of instantly discarding the objective function of (1), to consider a convex combination of it and $\Delta(x, \tilde{x})$, reducing gradually the influence of the objective term. The hope is that FP still converges to a feasible solution but it concentrates the search on the region of high-quality points. The modified objective function $\triangle_{\alpha}(x, \tilde{x})$ is defined as

$$
\triangle_{\alpha}(x, \tilde{x}):=(1-\alpha) \triangle(x, \tilde{x})+\alpha \frac{\|\Delta\|}{\|c\|} c^{T} x, \quad \alpha \in[0,1],
$$

where $\|\cdot\|$ is the Euclidean norm of a vector, and $\triangle$ is the objective function vector of $\triangle(x, \tilde{x})$ (i.e., at stage 1 is the number of binary variables, and at stage 2 is the number of integer (both general integer and binary) variables). At each FP iteration $\alpha$ is geometrically decreased with a fixed factor $\varphi<1$, i.e., $\alpha_{t+1}=\varphi \alpha_{t}$ and $\alpha_{0} \in[0,1]$. Notice that the original FP algorithm 
is obtained using $\alpha_{0}=0$. The objective FP algorithm is basically the same as the original FP algorithm of Figure 1, replacing $\Delta(x, \tilde{x})$ by $\triangle_{\alpha_{t}}\left(x^{*}, \tilde{x}\right)$ at line 5 , performing at the beginning the initialization of $\alpha_{0}$, and adding at the end of the loop $\alpha_{t+1}=\varphi \alpha_{t}$.

\section{The analytic center feasibility pump}

\subsection{The analytic center}

Given the LP relaxation (2), its analytic center is defined as the point $\bar{x} \in P$ that minimizes the primal potential function $-\sum_{i=1}^{n} \ln x_{i}$, i.e.,

$$
\begin{aligned}
\bar{x}=\arg \min _{x} & -\sum_{i=1}^{n} \ln x_{i} \\
\text { s. to } & A x=b \\
& x>0 .
\end{aligned}
$$

Note that constraints $x>0$ could be avoided, since the domain of $\ln$ are the positive numbers. Problem (7) is a linearly constrained strictly convex optimization problem. It is easily seen that the objective function $\min -\sum_{i=1}^{n} \ln x_{i}$ is equivalent to $\max \prod_{i=1}^{n} x_{i}$. Therefore, the analytic center provides the point that maximizes the distance to the hyperplanes $x_{i}=0, i=1, \ldots, n$, and it is thus expected to be well centered in the interior of the polytope $P$. We note that the analytic center is not a topological property of a polytope, and it depends on how $P$ is defined. In this sense, redundant inequalities may change the location of the analytical center. Additional details can be found in [13].

The analytic may be computed by solving the KKT conditions of (7)

$$
\begin{aligned}
A x & =b \\
A^{T} y+s & =0 \\
x_{i} s_{i} & =1 \quad i=1, \ldots, n \\
(x, s) & >0,
\end{aligned}
$$

$y \in \mathbb{R}^{m}$ and $s \in \mathbb{R}^{n}$ being the Lagrange multipliers of $A x=b$ and $x>0$ respectively. Alternatively, and in order to use an available highly efficient implementation, the analytic center was computed in this work by applying a primal-dual path-following interior-point algorithm to the barrier problem of (2), after removing the objective function term (i.e., setting $c=0$ ):

$$
\begin{array}{ll}
\min _{x} & -\mu \sum_{i=1}^{n} \ln x_{i} \\
\text { s. to } & A x=b \\
& x>0,
\end{array}
$$

where $\mu$ is a positive parameter (the parameter of the barrier) that tends to zero. The arc of solutions of (9) $x^{*}(\mu)$ is named the central path. The central path converges to the analytic center of the optimal set. When $c=0$ (as in (9)) the central path converges to the analytic center of the feasible set $P[13]$.

\subsection{Using the analytic center in the feasibility pump heuristic}

Once the analytic center has been computed, it can be used to (in theory infinitely) increase the number of feasible points candidates to be rounded. Instead of rounding, at each FP iteration, the feasible point $x^{*} \in P$, points on the segment

$$
x(\gamma)=\gamma \bar{x}+\left(\begin{array}{c}
1-\gamma) x^{*} \\
4
\end{array} \quad \gamma \in[0,1]\right.
$$




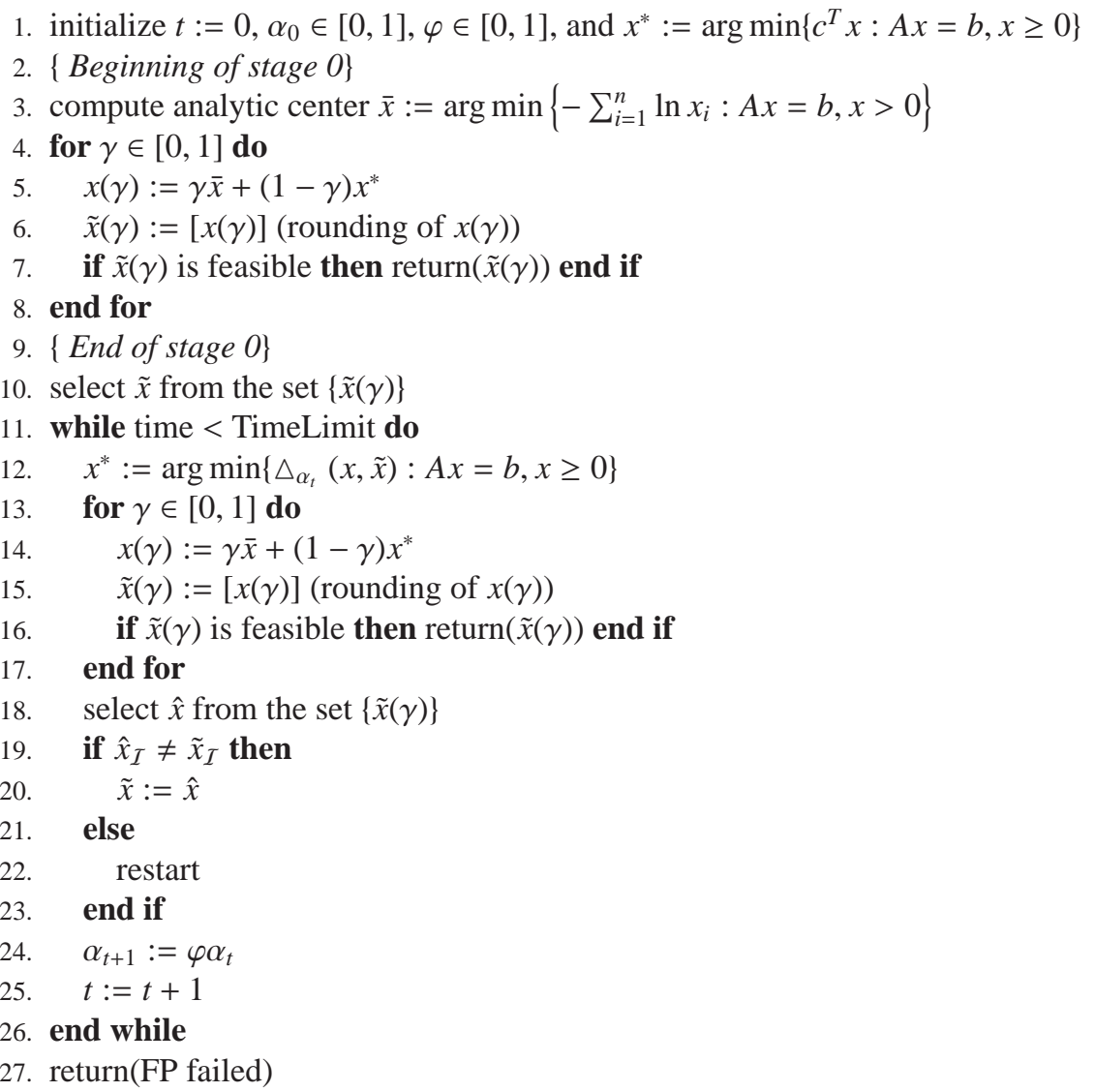

Figure 2: The analytic center feasibility pump heuristic 
will be considered. Note that the segment is feasible, since it is a convex combination of two feasible points.

The analytic center FP first considers a stage 0 (which is later applied at each FP iteration) where several $x(\gamma)$ points are tested, from $\gamma=0$ to $\gamma=1$ (i.e, from $x^{*}$ to $\bar{x}$ ). Each $x(\gamma)$ is rounded to $\tilde{x}(\gamma)$. If $\tilde{x}(\gamma)$ is feasible, then a feasible integer solution was found and the procedure is stopped at the stage 0 . Otherwise the algorithm proceeds with the next stage of FP, considering two different options:

a) using the point $\tilde{x}(0)=\left[x^{*}\right]$ (option $\left.\gamma=0\right)$;

b) using the point $\tilde{x}(\gamma)$ that minimizes $\|\tilde{x}(\gamma)-x(\gamma)\|_{\infty}$ (option $\left.L_{\infty}\right)$.

If the first option is applied at each FP iteration, and no feasible $\tilde{x}(\gamma)$ for $\gamma>0$ is found, the analytic center FP behaves as the standard FP algorithm. In the second option, if no feasible $\tilde{x}(\gamma)$ is found, the procedure selects the $x(\gamma)$ which is closer to $[x(\gamma)]$ according to the $L_{\infty}$ norm. The aim is to select the point with more chances to become both integer and feasible, in an attempt to reduce the number of FP iterations. This second option provided better results in general and it was used in the computational results of Section 3. It is worth to note that if the rounding of several $x(\gamma)$ points is feasible, the procedure selects the one with a lower $\gamma$, i.e., the one closer to $x^{*}$ (instead of the one closer to the analytic center $\bar{x}$ ), since this point was computed considering the objective function (for $\alpha>0$ ). An outline of the algorithm is shown in Figure 2.

\section{Computational results}

The analytic center FP was implemented using the base code of the objective FP, freely available from http: //www . or . deis . unibo.it/research_pages/ORcodes/FP-gen. html. The base FP implementation was extended for computing the analytic center using three different interior-point solvers, CPLEX [10], GLPK [9] and PCx [6]. The new code can be obtained from the authors on request. CPLEX integrates better with the rest of the FP code, which also relies on CPLEX, and it also turned out to be significantly more efficient than GLPK and PCx. On the other hand, even deactivating all the preprocessing options and removing the crossover postprocess, CPLEX was not always able to provide the analytic center of $P$ because of its aggressive reduced preprocessing (which can not be deactivated as we were told by CPLEX developers). For instance, for $P=\left\{x: \sum_{i=1}^{n}=n, x \geq 0\right\}$, the barrier option of CPLEX did not apply the interior-point algorithm, not providing an interior solution (i.e., it provided $x_{i}=n, x_{j}=0, j \neq i$ ), whereas both GLPK and PCx reported the right analytic center $x_{i}=1, i=1, \ldots, n$. Of the other two solvers, PCx turned out to be much more efficient than GLPK. Indeed, PCx may handle upper bounds implicitly (i.e., $0 \leq x \leq 1$ from linear relaxations of $x \in\{0,1\}$ ) in its interior-point implementation, whereas GLPK transforms the problem to the standard form (replacing $x \leq 1$ by $x+s=1, s \geq 0$ ), significantly increasing the size of the Newton's system to be solved at each interior-point iteration.

The analytic center FP implementation was applied to a subset of MIPLIB2003 instances, whose dimensions are shown in Table 1. Columns "rows", "cols", "nnz", "int", "bin" and "con" provide respectively the number of constraints, variables, nonzeros, general integer variables, binary variables, and continuous variables of the instances. Column "objective" shows the optimal objective function. Unknown optimal objectives are marked with a "?".

Table 2 shows the results obtained for the objective FP, the analytic center FP using PCx, and the analytic center FP using CPLEX-12.1. For each variant, Table 2 reports the number of FP iterations (columns "niter"), objective value of feasible point found ("fobj"), gap between 


\begin{tabular}{|c|c|c|c|c|c|c|c|}
\hline Instance & rows & cols & $\mathrm{nnz}$ & int & bin & con & objective \\
\hline 10teams & 230 & 2025 & 12150 & 0 & 1800 & 225 & 924 \\
\hline alcls1 & 3312 & 3648 & 10178 & 0 & 192 & 3456 & 11503.40 \\
\hline aflow30a & 479 & 842 & 2091 & 0 & 421 & 421 & 1158 \\
\hline aflow $40 \mathrm{~b}$ & 1442 & 2728 & 6783 & 0 & 1364 & 1364 & 1168 \\
\hline air04 & 823 & 8904 & 72965 & 0 & 8904 & 0 & 56137 \\
\hline air05 & 426 & 7195 & 52121 & 0 & 7195 & 0 & 26374 \\
\hline arki001 & 1048 & 1388 & 20439 & 96 & 415 & 877 & 7580810 \\
\hline atlanta-ip & 21732 & 48738 & 257532 & 106 & 46667 & 1965 & 90.00 \\
\hline cap6000 & 2176 & 6000 & 48243 & 0 & 6000 & 0 & -2451380 \\
\hline dano3mip & 3202 & 13873 & 79655 & 0 & 552 & 13321 & \\
\hline danoint & 664 & 521 & 3232 & 0 & 56 & 465 & 65.66 \\
\hline disctom & 399 & 10000 & 30000 & 0 & 10000 & 0 & -5000 \\
\hline $\mathrm{ds}$ & 656 & 67732 & 1024059 & 0 & 67732 & 0 & 93.52 \\
\hline fast0507 & 507 & 63009 & 409349 & 0 & 63009 & 0 & 174 \\
\hline fiber & 363 & 1298 & 2944 & 0 & 1254 & 44 & 405935 \\
\hline fixnet6 & 478 & 878 & 1756 & 0 & 378 & 500 & 3983 \\
\hline gesa2-o & 1248 & 1224 & 3672 & 336 & 384 & 504 & 25779900 \\
\hline gesa2 & 1392 & 1224 & 5064 & 168 & 240 & 816 & 25779900 \\
\hline glass4 & 396 & 322 & 1815 & 0 & 302 & 20 & 1200010000 \\
\hline harp2 & 112 & 2993 & 5840 & 0 & 2993 & 0 & -73899800 \\
\hline liu & 2178 & 1156 & 10626 & 0 & 1089 & 67 & \\
\hline manna81 & 6480 & 3321 & 12960 & 3303 & 18 & 0 & -13164 \\
\hline markshare1 & 6 & 62 & 312 & 0 & 50 & 12 & 1 \\
\hline markshare2 & 7 & 74 & 434 & 0 & 60 & 14 & 1 \\
\hline mas74 & 13 & 151 & 1706 & 0 & 150 & 1 & 11801.20 \\
\hline $\operatorname{mas} 76$ & 12 & 151 & 1640 & 0 & 150 & 1 & 40005.10 \\
\hline misc07 & 212 & 260 & 8619 & 0 & 259 & 1 & 2810 \\
\hline $\mathrm{mkc}$ & 3411 & 5325 & 17038 & 0 & 5323 & 2 & -563.84 \\
\hline $\bmod 011$ & 4480 & 10958 & 22254 & 0 & 96 & 10862 & -54558500 \\
\hline modglob & 291 & 422 & 968 & 0 & 98 & 324 & 20740500 \\
\hline msc98-ip & 15850 & 21143 & 92918 & 53 & 20237 & 853 & 19839500 \\
\hline mzzv11 & 9499 & 10240 & 134603 & 251 & 9989 & 0 & -21718 \\
\hline mzzv42z & 10460 & 11717 & 151261 & 235 & 11482 & 0 & -20540 \\
\hline net12 & 14021 & 14115 & 80384 & 0 & 1603 & 12512 & 214 \\
\hline noswot & 182 & 128 & 735 & 25 & 75 & 28 & -41 \\
\hline nsrand-ipx & 735 & 6621 & 223261 & 0 & 6620 & 1 & 51200 \\
\hline nw04 & 36 & 87482 & 636666 & 0 & 87482 & 0 & 16862 \\
\hline opt1217 & 64 & 769 & 1542 & 0 & 768 & 1 & -16 \\
\hline p2756 & 755 & 2756 & 8937 & 0 & 2756 & 0 & 3124 \\
\hline pk1 & 45 & 86 & 915 & 0 & 55 & 31 & 11 \\
\hline pp08aCUTS & 246 & 240 & 839 & 0 & 64 & 176 & 7350 \\
\hline pp08a & 136 & 240 & 480 & 0 & 64 & 176 & 7350 \\
\hline protfold & 2112 & 1835 & 23491 & 0 & 1835 & 0 & -31 \\
\hline qiu & 1192 & 840 & 3432 & 0 & 48 & 792 & -132.87 \\
\hline roll3000 & 2295 & 1166 & 29386 & 492 & 246 & 428 & 12890 \\
\hline rout & 291 & 556 & 2431 & 15 & 300 & 241 & 1077.56 \\
\hline setlch & 492 & 712 & 1412 & 0 & 240 & 472 & 54537.80 \\
\hline seymour & 4944 & 1372 & 33549 & 0 & 1372 & 0 & 423 \\
\hline $\begin{array}{l}\text { sp97ar } \\
\text { s. }\end{array}$ & 1761 & 14101 & 290968 & 0 & 14101 & 0 & 660706000 \\
\hline swath & 884 & 6805 & 34965 & 0 & 6724 & 81 & 467.40 \\
\hline timtab1 & 171 & 397 & 829 & 94 & 64 & 239 & 764772 \\
\hline timtab2 & 294 & 675 & 1482 & 164 & 113 & 398 & 1096560 \\
\hline $\operatorname{tr} 12-30$ & 750 & 1080 & 2508 & 0 & 360 & 720 & 130596 \\
\hline vpm2 & 234 & 378 & 917 & 0 & 168 & 210 & 13.75 \\
\hline
\end{tabular}

Table 1: Characteristics of the subset of MILP instances from MIPLIB 2003 


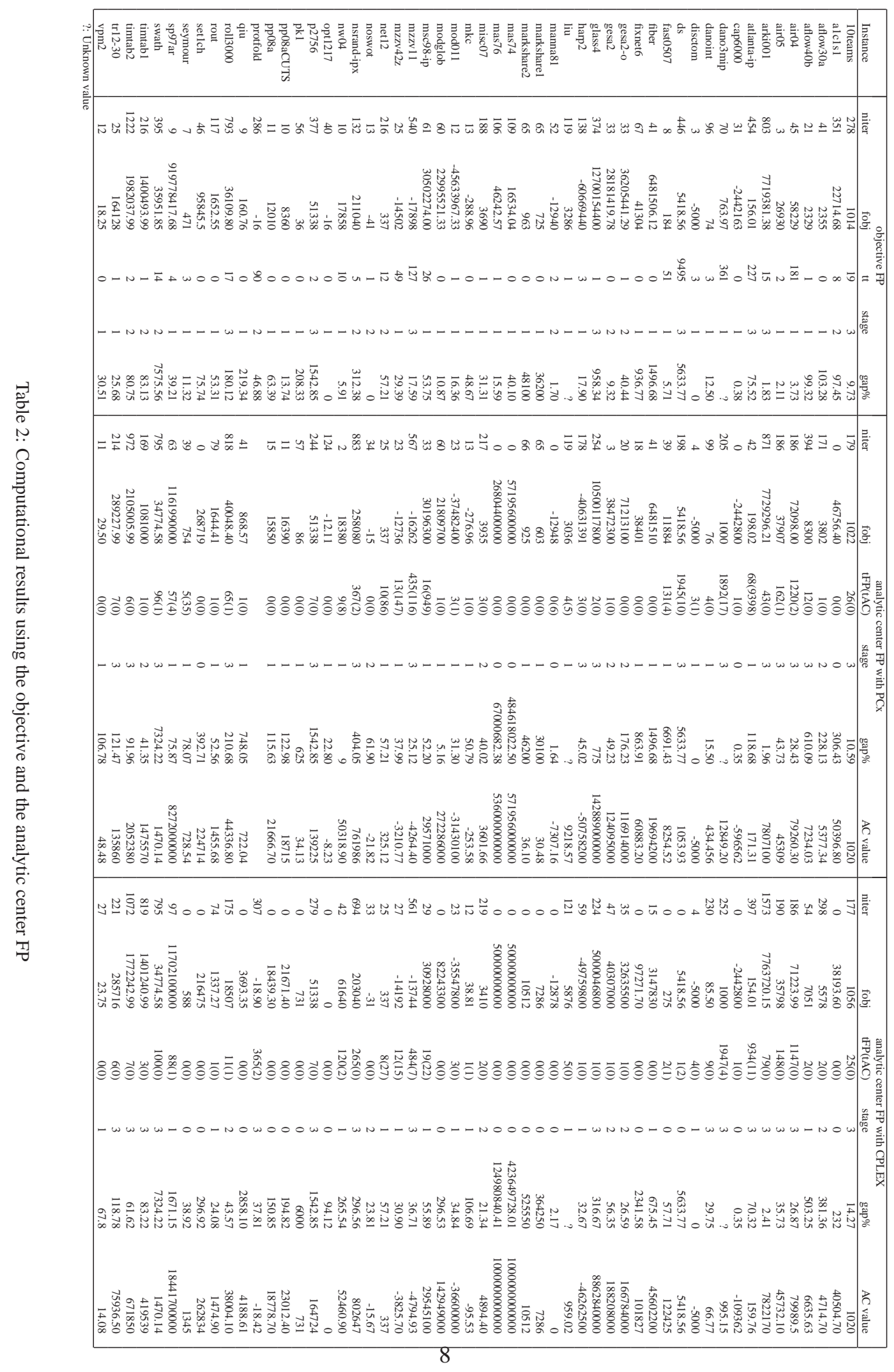


the feasible and the optimal solution ("gap\%"), and FP stage where the feasible point was found ("stage"). For the objective FP, the column "tt" shows the total CPU time. For the two analytic center FP variants, columns "tFP(tAC))" report separately the CPU time spent in stages 1 to 3 ("tFP") and the time for computing the analytic center at stage 0 (in brackets, "tAC"); the total time is the sum of the two values. For the two analytic center FP variants columns "AC value" show the value of the original objective function evaluated at the analytic center. Differences are due to different computed analytic centers because both solvers apply very distinct preprocessing strategies. The default FP settings were used as suggested in [1]. All runs were carried on a Dell PowerEdge 6950 server with four dual core AMD Opteron $82223.0 \mathrm{GHZ}$ processors (without exploitation of parallelism capabilities) and 64 GB of RAM.

Although from Table 2, in general it can be concluded that the analytic center FP is inferior to the objective FP, there are some notable exceptions. For instance, for the 13 instances with both binary and general integer variables, the analytic center FP (either with PCx or CPLEX) obtained a solution with a lower gap than the objective FP in eight of the 13 instances; in some cases more efficiently and even being able to find a solution when the objective FP failed (i.e., it required stage 3), as for instances "roll3000" and "atlanta-ip" (in this latter case, however, at the expense of a very large CPU time). On the other hand, for problems with only binary variables the analytic center FP obtained solutions with a lower gap in very few instances. A possible explanation of this different behaviour in problems with and without general integer variables is that, for a binary problem, the only feasible integer points "close" to the segment $x(\gamma)$ are $\{0,1\}^{n}$, which in addition may be far from the center. For problems with general integer variables, the number of feasible integer solutions close to the analytic center will be, in general, much larger. For some problems with only integer binary variables, the analytic center FP behaved very poorly, as for "mas74" and "mas76" (it stopped at stage 0 in those cases). However, in other instances it was much more efficient obtaining the same gap that the objective FP, as for "ds". Note that for "ds" the analytic center FP with CPLEX obtained the feasible solution in one second at stage 0 (the other two variants failed, requiring stage 3 ). However, in that case CPLEX did not really compute the analytic center: it solved $\min _{x}\{0: x \in P\}$ heuristically, instead of applying the barrier algorithm, as required. It thus considered a segment between two feasible solutions, none of them being the analytic center of $P$. Therefore, the idea of using a segment of feasible points is not restricted to the case where one of the endpoints is the analytic center, and it can be extended to more general situations.

\section{Conclusions}

The analytic center FP is an extension of the original FP where candidate points to be rounded are found in a segment of feasible points, one of the extremes being the analytic center. The objective FP is a particular case where the endpoint associated to the solution of the relaxed problem is selected as the point to be rounded. The analytic center FP has not been shown to outperform the objective FP, in general. However for problems with both general integer and binary variables, and for some particular binary problems, it may result in more efficient and lower gap solutions. The analytic center FP could also be used with the recent rounding scheme based on constraint propagation suggested in [8]. 


\section{Acknowledgments}

This work has been supported by grants MTM2009-08747 of the Spanish Ministry of Science and Innovation, and SGR-2009-1122 of the Government of Catalonia.

\section{References}

[1] T. Achterberg, T. Berthold, Improving the feasibility pump, Discrete Optimization 4 (2007), 77-86.

[2] T. Achterberg, G. Gamrath, T. Koch, A. Martin, The mixed integer programming library: MIPLIB 2003. http://miplib.zib.de .

[3] H.Y. Benson, Mixed integer nonlinear programming using interior-point methods, Optimization Methods and Software (2010), doi: 10.1080/10556781003799303.

[4] P. Bonami, L.T. Biegler, A.R. Conn, G. Cornuejols, I.E. Grossman, C.D. Laird, J. Lee, A. Lodi, F. Margot, N. Sawaya, A.Wachter, An algorithmic framework for convex mixed integer nonlinear programs, Discrete Optimization 5 (2008), 186-204

[5] L. Bertacco, M. Fischetti, A. Lodi, A feasibility pump heuristic for general mixed-integer problems, Discrete Optimization 4 (2007), 63-76.

[6] J.Czyzyk, S. Mehrotra, M. Wagner, S.J. Wright, PCx: an interior-point code for linear programming, Optimization Methods and Software 11 (1999) 397-430.

[7] M. Fischetti, F. Glover, A. Lodi, The Feasibility Pump, Mathematical Programming 104 (2005), 91-104.

[8] M.Fischetti, D. Salvagnin, Feasibility pump 2.0, Mathematical Programming Computation 1 (2009), 201-222.

[9] GNU, GNU Linear Programming Kit v. 4.43, 2010.

[10] IBM ILOG CPLEX 12.1, User's Manual, 2010.

[11] J.E. Mitchell, Fixing variables and generating classical cutting planes when using an interior point branch and cut method to solve integer programming problems, European Journal of Operational Research 97 (1997), 139-148.

[12] J.E. Mitchell, M.J. Todd, Solving combinatorial optimization problems using Karmarkar's algorithm, Mathematical Programming 56 (1992), 245-284.

[13] Y. Ye, Interior Point Algorithms. Theory and Analysis, Wiley, 1997. 